\title{
Data reduction software for the Gemini high resolution optical spectrograph
}

Michael J. Ireland, Marc White, Joao P. Bento, Tony Farrell, Kathleen Labrie, et al.

Michael J. Ireland, Marc White, Joao P. Bento, Tony Farrell, Kathleen Labrie, Lance Luvaul, Jon G. Nielsen, Chris Simpson, "Data reduction software for the Gemini high resolution optical spectrograph," Proc. SPIE 10707, Software and Cyberinfrastructure for Astronomy V, 1070735 (6 July 2018); doi: 10.1117/12.2314418

Event: SPIE Astronomical Telescopes + Instrumentation, 2018, Austin, Texas, United States 


\title{
Data reduction software for the Gemini high resolution optical spectrograph
}

\author{
Michael J. Ireland ${ }^{\mathrm{a}}$, Marc White ${ }^{\mathrm{a}}$, Joao P. Bento ${ }^{\mathrm{a}}$, Tony Farrell ${ }^{\mathrm{c}}$, Kathleen Labrie ${ }^{\mathrm{b}}$, Lance \\ Luvaul $^{\mathrm{a}}$, Jon G. Nielsen ${ }^{\mathrm{a}}$, and Chris Simpson ${ }^{\mathrm{b}}$ \\ ${ }^{a}$ Research School of Astronomy and Astrophysics, Australian National University, Canberra, \\ ACT 2611, Australia \\ ${ }^{\mathrm{b}}$ Gemini Observatory, Hilo, HI 96720, USA \\ cAustralian Astronomical Observatory, 105 Delhi Rd, North Ryde, NSW 2113, Australia
}

\begin{abstract}
The data reduction software for the Gemini High Resolution Optical SpecTrograph (GHOST) presents a number of unusual challenges. Star light from one or two objects and simultaneous sky is collected in integral field units rather than a slit or well-separated fibers. When used with binning, individual fibers are not resolved, and the optimal pixel weighting is derived from a simultaneous slit viewing camera. We describe the data reduction approach taken, including testing using an optical physics-based data simulator, and an object-oriented and modular approach to spectral extraction that fits within the Gemini recipe system, DRAGONS, using AstroConda.
\end{abstract}

Keywords: Astronomical data reduction, high resolution spectroscopy, precision radial velocity, echelle spectroscopy

\section{INTRODUCTION}

The Gemini High-Resolution Optical SpecTrograph (GHOST) is optimised for efficiency in reference seeing conditions of 0.8 arcsec. $^{1}$ Both detector pixels and instrument étundue are used highly efficiently, so that a spectral resolving power of $>75,000$ (high resolution, single-object mode) or $>50,000$ (standard resolution, dualobject mode) is possible using a single $400 \mathrm{~mm}$ long grating and 2 arms (one $6 \mathrm{k} \mathrm{x} 6 \mathrm{k}$ and one $4 \mathrm{k} \mathrm{x} 4 \mathrm{k}$ ). The stellar image is sliced into 7 (standard resolution) or 19 (high resolution) components, and re-formatted into a slit with no gaps between fiber images for a single star, and no gaps between adjacent fiber images of a target and sky. Furthermore, the requirement for spectroscopy of very faint stars, for e.g. elemental abundances of stars in dwarf galaxies, means that binning modes of up to 8 pixels are needed. This means that it is not possible to extract individual fibers, and a custom pipeline with some novel algorithms is needed. Furthermore, GHOST has the ability to simultaneously image the slit profile. This is a powerful tool for accurate spectral extraction, but also produces software challenges.

\section{SOFTWARE REQUIREMENTS}

\subsection{Science Requirements}

The main requirements derived from the science requirements are that the pipeline works for all defined modes (Table 1) and provides a near-optimal (i.e. science quality), mathematically-correct reduction. In turn, this requires the ability to take into account the slit profile to improve the signal-to-noise in good seeing, requires accurate but optional sky subtraction (to a goal of $1 \%$ relative accuracy), with a final output that includes barycentric correction, and the combination of adjacent orders on a fixed wavelength grid.

The scope of the initial GHOST data reduction software is to provide a Gemini-compliant pipeline capable of near-optimal spectra extraction to science-usable flux versus wavelength. This includes an extraction able to meet the $10 \mathrm{~m} \mathrm{~s}^{-1}$ radial velocity precision science requirement, with the software in principle being extensible to attempt to meet the $1 \mathrm{~m} \mathrm{~s}^{-1}$ goal (depending also on e.g. the spectrograph exceeding requirements and meeting goals). However, the data reduction software does not include the complete radial velocity pipeline.

Further author information: M.J.I.: E-mail: michael.ireland@anu.edu.au 
Table 1. Science modes of GHOST

\begin{tabular}{|c|c|c|c|}
\hline Configuration & Fiber bundles & Resolution & Detector Binning \\
\hline Two-Target & $\begin{array}{l}\text { low-res bundle on IFU1 } \\
\text { low-res sky on IFU1 } \\
\text { low-res bundle on IFU2 }\end{array}$ & $50 \mathrm{k}$ & $1 \times 2$ \\
\hline Two-Target Faint & $\begin{array}{l}\text { low-res bundle on IFU1 } \\
\text { low-res sky on IFU1 } \\
\text { low-res bundle on IFU2 }\end{array}$ & $50 \mathrm{k}$ & $2 \times 4$ \\
\hline Beam-Switching & $\begin{array}{l}\text { low-res bundle on IFU1 } \\
\text { low-res sky on IFU1 } \\
\text { low-res bundle on IFU2 }\end{array}$ & $50 \mathrm{k}$ & $1 \times 2$ \\
\hline Beam-Switching Faint & $\begin{array}{l}\text { low-res bundle on IFU1 } \\
\text { low-res sky on IFU1 } \\
\text { low-res bundle on IFU2 }\end{array}$ & $50 \mathrm{k}$ & $2 \times 8$ \\
\hline High Resolution & $\begin{array}{l}\text { high-res bundle on IFU1 } \\
\text { high-res sky on IFU2 }\end{array}$ & $75 \mathrm{k}$ & $1 \times 1$ \\
\hline High Resolution Faint & $\begin{array}{l}\text { high-res bundle on IFU1 } \\
\text { high-res sky on IFU2 }\end{array}$ & $75 \mathrm{k}$ & $1 \times 8$ \\
\hline High Resolution PRV & $\begin{array}{l}\text { high-res bundle on IFU1 } \\
\text { high-res sky on IFU2 } \\
\text { high-res ThXe }\end{array}$ & $75 \mathrm{k}$ & $1 \times 1$ \\
\hline
\end{tabular}

\subsection{Gemini Requirements}

Data processing for Gemini is undergoing a process of modernisation within the framework of DRAGONS (Data Reduction for Astronomy Gemini Observatory North South; https://github.com/GeminiDRSoftware/DRAGONS). The data reduction software for GHOST is the first instrument pipeline to be specifically developed for DRAGONS.

\subsubsection{Computing Environment}

The GHOST Data Reduction (GHOSTDR) pipeline, and the wider DRAGONS system, is designed to run within a Gemini-supplied AstroConda environment*, which is a PYTHON distribution with specific packages supplied. PYTHON 2.7 is the recommended version for running DRAGONS and GHOSTDR, although the code is designed to be compatible with PyThon 3. AstroConda provides all the necessary packages for astronomical scientific computing (e.g., NumPy, ScIPy, AstroPy, etc.) For compatibility and ease-of-use reasons, we restrict ourselves to only using those Python packages which are shipped with the Gemini AstroConda environment. This is particularly important because DRAGONS is a multi-use system. It is designed to handle both near-real-time data quality assurance at telescope sites, and as a one-stop shop for science-level data reduction across a variety of computing environments. Therefore, DRAGONS packages must be able to run wherever the standard Gemini AstroConda environment is deployed.

\subsubsection{DRAGONS Overview}

The core component of DRAGONS is the RecipeSystem. The functionality of the RecipeSystem is provided by the Reduce class and the associated reduce script, which provide an application programming interface (API) and command-line interface to the system, respectively. With minimal input from the user, the RecipeSystem is able to identify the source and type of the data being reduced, determine the correct reduction scheme, perform the reduction, and write the output to disk.

Each instrument supported by DRAGONS, including GHOST, is required to provide a set of primitive classes, within which are defined class methods known as primitives. Each primitive defines a single operation corresponding to a step in the instrument's data flow (e.g., §3.2). Primitives may be applicable to all observation types (e.g., bias correction), or customized to a specific subset of observation types (e.g., profile extraction is

\footnotetext{
*http://www.gemini.edu/node/12665
} 
only applicable to GHOST frames when the fibres are illuminated). For each observation type (i.e., bias, dark, flat, etc.), primitives are grouped into functions called recipes, which executes the primitives in sequence on every member of the input data set. Recipes are grouped into modes, describing the intended use of the recipes. The two primary modes are the Quality Assurance mode (for near-real-time data quality monitoring), and the Science Quality mode (for the generation of publication-ready data).

To relate data to recipes, an instrument pipeline defines a collection of tags. Data receive a tag set based on the presence/absence and/or values of certain FITS header keywords. Recipes are associated with data based on the tag set; if the recipe to use is not explicitly specified, the RecipeSystem will automatically select the appropriate recipe to run, by comparing the input data tag set with the target tag set specified in each recipe.

Data is handled within DRAGONS using the AstroData package, which implements the AstroData class. Each instrument defines a set of methods on the AstroData class called descriptors, which provide an instrumentagnostic way of accessing important FITS headers. AstroData provides a standard API for accessing and modifying data, regardless of instrument.

\section{GHOSTDR IMPLEMENTATION}

\subsection{Implementation within DRAGONS}

The data reduction pipeline for GHOST, GHOSTDR, is currently being implemented within the DRAGONS framework. We are working in concert with the ongoing development of the DRAGONS system by Gemini.

DRAGONS packages are required to be PEP 8 compliant $^{\dagger}$, and we use the pylint tool ${ }^{\ddagger}$ to validate that our code satisfies this requirement. Unit tests are also a requirement, and are evaluated using the pytest tool ${ }^{\S}$. Test suites are currently under construction. The code is heavily documented, and we make significant use of the autodoc functionality within the SPHINx documentation system ${ }^{\top}$ to generate User's and Programmer's Manuals. We are careful to ensure that code is correctly documented as it is being written.

Static configuration items (e.g., arc line lists, initial spectrograph profile models, etc.) are stored within DRAGONS' lookups architecture. Specialised helper functions are included to find the appropriate product from the lookups system; e.g., a function is provided to locate the correct initial profile model (§3.5.6) based on the requesting data's spectrograph arm and exposure epoch. This system also holds important static information for GHOSTDR, such as timestamp header keywords and default header keyword comments.

GHOSTDR uses standard DRAGONS calls to the Gemini Calibration Manager to store and retrieve processed calibration images (i.e., biases, darks, flats, arc and standards). The Calibration Manager is an internal component of the main Gemini Data Archive used for facility operations, and has access to all archival Gemini calibration products. A local Calibration Manager for end-users is currently under development. The local Manager uses the same calibration rules to associate science data to calibrators, but a different (local) database and access layer, and will only have access to calibration data loaded into it by the local user as a result of reducing calibration exposures. The necessary calibration observations will be packaged with science observations as associated calibrations in the archive. GHOSTDR is being undertaken using an unpublished beta version of the local Calibration Manager, and standard star spectra are currently handled separately to the DRAGONS system.

\subsection{Data Flow}

Figure 1 graphically describes the data reduction flow for the GHOST instrument. Figure 2 shows the order in which GHOST data are reduced, grouped by data type. We elaborate further on this process below.

\footnotetext{
${ }^{\dagger}$ https://www.python.org/dev/peps/pep-0008/

${ }^{\ddagger}$ https://www.pylint.org/

$\S$ https://docs.pytest.org

『 http://www.sphinx-doc.org/
} 
GHOST Data Processing Flowchart



Figure 1. Flowchart describing the data reduction flow for the GHOST instrument. 


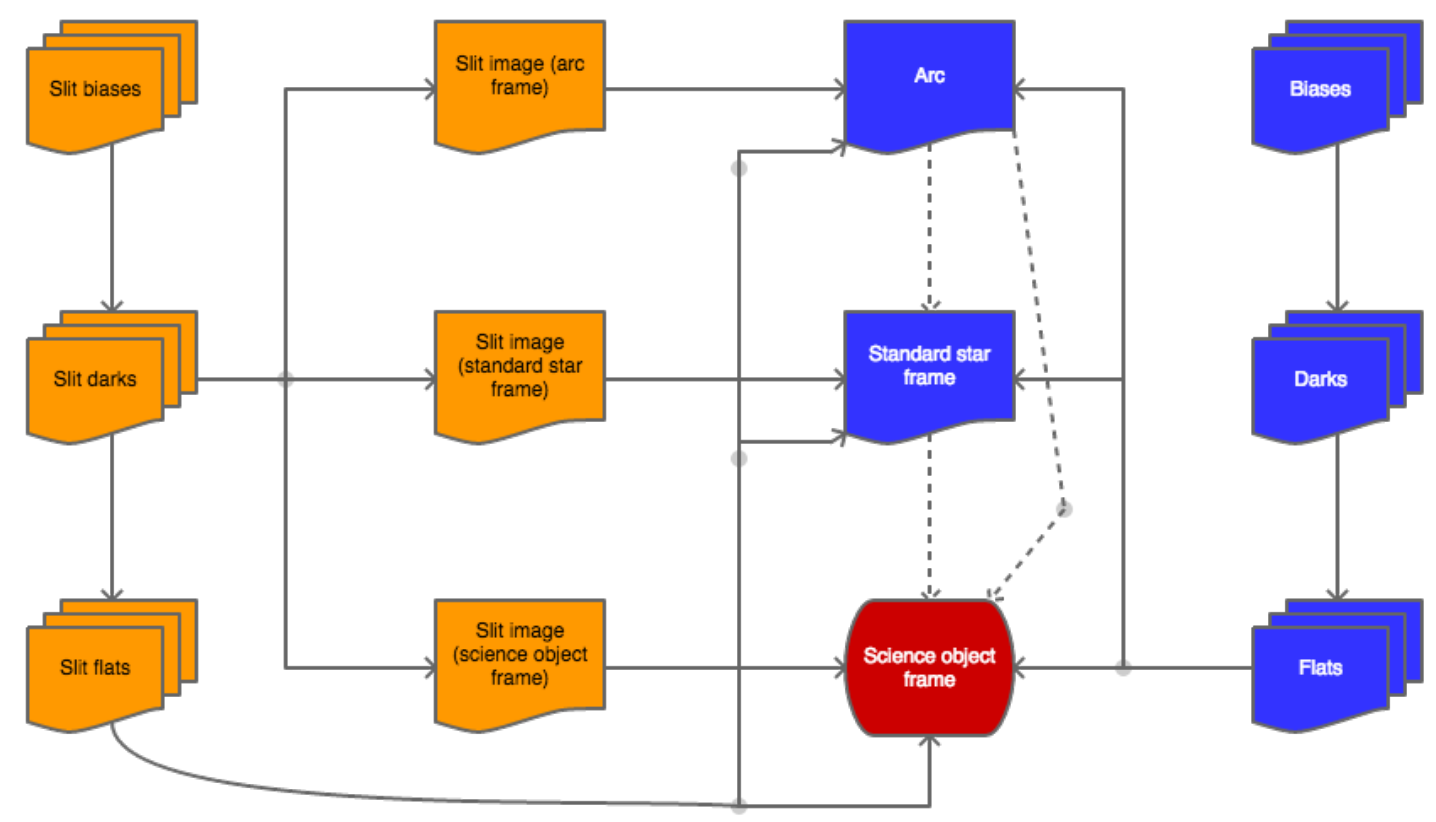

Figure 2. Data Reduction Sequence for GHOST Data. Dashed arrows indicate optional data reduction steps.

\subsection{Slit Viewer Reduction}

Data from the slit viewer camera are processed in a reasonably conventional way for an imaging camera system. The recipe first bias and dark corrects the data, and adds data quality and variance planes based on a poisson plus read noise model of the detector. Cosmic rays are found by searching for outliers on a pixel-by-pixel basis, where outliers are defined in terms of a multiple (defaulting to 20) of the median absolute deviation. These pixels are replaced with the median over all frames. This algorithm requires at least 3 slit viewing frames per science data set, which is the case for any long $(>30 \mathrm{~s})$ science exposure sequence where the probability of a cosmic ray is non-negligible. From the set of slit viewer frames, two key outputs are produced: the mean exposure epoch, and the stacked slit viewer frame for each science frame. The mean exposure epoch is the epoch for which a barycentric correction should be computed, accounting for changes in seeing or transparency during an exposure. The stacked slit viewer frame is used to determine the object profile. For both computations, slit viewer frames that do not have exposures fully overlapping with the science exposures are downweighted linearly according to their overlap percentage.

\subsection{Calibration Frames}

Bias and dark frames are reduced in the standard fashion. Stacked dark frames are sigma-clipped before they are stored (with a default sigma value of 3). Bias and dark reduction is performed on an amplifier-wise basis.

Initial flat field processing is as per the standard fashion. The individual amplifier extensions are then tiled together into a single homogeneous frame, and then the concurrent slit viewer exposures are used to determine the flat field profile.

\subsection{Science Data Reduction}

\subsubsection{Weighted Extraction}

In any spectrograph extraction routine, pixels in the direction orthogonal to the wavelength axis have to be averaged or summed in order to maximise the signal-to-noise ratio. We will call this direction the $x$-direction, 
as the physical slit is horizontal in the instrument laboratory frame. In the case of multi-fiber spectroscopy, where there is some overlap between neighboring fibers, the optimal algorithm is described in Sharp and Birchall $(2010) .^{2}$

For GHOST, the situation is a somewhat more complex, because the "object profile" is not fixed as is for the case of single-fiber spectroscopy. Each object has a profile that is measured from the slit viewing camera, which has to be scaled in the spectrograph $x$-direction (and also in flux) in order to match the data.

We will first consider the profiles for object and sky along a single order. These profiles, normalised to 1 along the $x$-direction, are given by $\phi_{k i}$ for object (or sky) $k$, and $x$-direction pixel $i$. From the variance plane $\left(\sigma_{i}^{2}\right)$ of the data, we obtain weights for each pixel:

$$
w_{i}=\frac{1}{\sigma_{i}^{2}},
$$

with $w_{i}=0$ for bad pixels, including cosmic ray locations.

These weights are used to construct the following two matrices. First, the cross-talk matrix between profiles, which should be diagonal except for the (very large!) cross-talk between sky and object:

$$
C=c_{k j}=\Sigma_{i} \phi_{k i} \phi_{j i} w_{i},
$$

and the naive extraction matrix :

$$
B=b_{k j}=\phi_{k j} w_{j} .
$$

In the case of no cross-talk, this extraction matrix would left multiply the optimally weighted pixel values $\mathbf{d}=D_{i}$ to form a maximum likelihood estimator for the true flux $\eta_{k}$ :

$$
\eta_{k}=\frac{\Sigma_{i} \phi_{k i} w_{i} D_{i}}{\Sigma_{i} \phi_{k i}^{2} w_{i}}=\frac{B \cdot \mathbf{d}}{\Sigma_{i} \phi_{k i}^{2} w_{i}} .
$$

In the presence of cross-talk, the simple division on the right hand side of this equation is not possible, and we aim to solve:

$$
C \cdot \eta=B \cdot \mathbf{d},
$$

which is solved by

$$
\eta_{k}=\Sigma_{i} z_{k i} D_{i}
$$

for an extraction weights matrix $Z=z_{k i}$ being computed by

$$
Z=C^{-1} \cdot B \text {. }
$$

This matrix also subtracts the sky as measured. This may not always be signal-to-noise optimal. For example, if emission from the sky or object 2 is negligible, object 1 is best extracted by ignoring the sky and object 2 . For this reason, data are extracted in modes corresponding to models with 1, 2 and 3 objects (including sky), so that the user can choose the most appropriate data product for their science.

The extraction weights, $z_{k j}$, are stored in an array that is $n_{x} \times n_{y}$ pixels in size, where weights from every order are added together. This method of storing the weights assumes no overlap in weights between orders otherwise the weights from neighboring orders would be saved on the same pixels. This is a problem for $8 \mathrm{x} 1$ binning in the shortest-wavelength orders, where there is overlap (at the level of up to 1 binned pixel) between neighboring orders. In this case, it is essential that these pixels are marked as bad in the reduction in both orders, to avoid cross-talk between orders. 


\subsubsection{Flat Field Correction}

There are two primary algorithms for flat field correction in spectroscopy: a long slit flat field, and an extracted fiber profile flat field. GHOST is in an intermediate regime, where the object profile varies from object to object and from object to flat lamp (due to different fibers being illuminated), yet is stable in terms of slit to pixel mapping. The primary flat field algorithm is similar to a long-slit flat field. During flat field processing, a model flat profile $\phi_{k}$ is constructed for every pixel $k$ for each of the red and blue cameras. This profile is normalised to 1.0 for every spectral direction pixel and every order. Note that we do not include a per-object index here, as the flat lamp can only illuminate the input fibers in the same way as the sky. The median combined flat field normalised by dividing by the median for all illuminated pixels, with no normalisation from order to order.

Prior to extraction, science data are corrected by the flat field as follows:

$$
D_{k}=\frac{r_{k} \phi_{k}}{f_{k}} . .
$$

Here $f_{k}$ is the normalised flat field, $r_{k}$ is the raw data and $\phi_{k}$ the flat field slit profile model. Note that the $\mathrm{Th} / \mathrm{Xe}$ simultaneous reference lamp is not flat-field corrected using this algorithm, with those pixels treated separately. The Th/Xe lamp is only extracted in 1x1 binning mode for precision radial velocity observations.

\subsubsection{Slit Tilt and PSF Variation Effects}

One problem with the algorithm above is that it assumes that the two-dimensional point-spread-function (PSF) is the product of spectral and spatial point spread functions. This is the case for single-fiber profiles imaged with spectrographs with typical aberrations as used by Sharp and Birchall, producing a near-Gaussian profile. However, for GHOST, even individual fiber profiles are not quite Gaussian. We avoid the most significant problems with this while preserving the need to weight differently at high versus low signal-to-noise by convolving the variance array both spatially and spectrally. In the current implementation, the spectral weights are convolved by a Hanning window of $\sim 7$ pixels FWHM, and the spatial variance is convolved by a window function of $\sim 2$ pixels FWHM.

\subsubsection{Weighted Extraction Principle in 2D}

For object profiles in two dimensions, i.e. with a small amount of slit tilt, we choose to extract as in one dimension, but we interpolate linearly between the nearest two pixels in the wavelength direction only. This results in the effective wavelength extracted for each pixel in the $x$-direction (along the slit) corresponds to the wavelength solution wavelength for the slit central pixel. This two dimensional weighting means that we add an additional dimension to the extraction weights variables:

$$
\eta_{k}=\Sigma_{l} \Sigma_{i} z_{k i l} a_{i l} D_{i},
$$

where the sum over the new index $l$ is over the pixels in the dispersion direction, and the dispersion interpolation weights are given by $a_{i l}$. As mentioned above, $a_{i l}$ is non-zero for only 2 pixels.

\subsubsection{The Polynomial Spectrograph Model}

The GHOST data reduction software relies heavily on a polynomial principle for all modelling of the spectrographs characteristics from the data. These include the location of the orders, the wavelength scale, and the reciprocal model that converts the sampled slit from the slit viewer to its image on the spectrograph CCD.

The idea is that, instead of a traditional empirical extraction (where the orders are scanned for their location and arc lines are detected blindly), we form a model of the spectrograph and fit the parameters using the flat fields and arcs to measure small changes in the spectrograph on a nightly basis. Then, the extraction process becomes relatively trivial with knowledge of where all the flux is, and the wavelength scale is uniquely determined. A major advantage of this method is that the entire spectrograph is modelled as one cohesive unit, instead of each individual order being treated as a separate entity. Ultimately, this means measurements such as apparent radial velocity shifts due to spectrograph drift can be determined using a single varying parameter, as opposed to a combination of measured shifts in all orders. 
The approach implemented is that of a sum of polynomials of polynomials. This differs from an approach where each physical parameter of the spectrograph is modelled individually, and focusses on a series of coefficients that represent various aspects of the CCD images. We thereby minimise the number of required parameters that describe the data.

The polyfit module within the GHOSTDR pipeline produces and manipulates files containing polynomial coefficients, where each row of the table are the coefficients for the polynomials as a function of order. These are then combined as a function of $y$-position on the CCD chip, where $y$ is defined as the CCD pixel numbers in the spectral direction.

For mathematical convenience and correspondence with an easily-identified reference, the polynomials are evaluated with respect to:

- a reference order, $m_{\text {ref }}$, defaulting to whatever order number is in the middle of the range used for each arm; and,

- the middle pixel on the chip, $y_{\text {middle }}$.

The functional form is:

$$
F(p)=p_{0}(m)+p_{1}(m) * y^{\prime}+p_{2}(m) * y^{\prime 2}+\ldots
$$

with $y^{\prime}=y-y_{m i d d l e}$, and:

$$
p_{0}(m)=q_{00}+q_{01} * m^{\prime}+q_{02} * m^{\prime 2}+\ldots
$$

with $m^{\prime}=m_{\text {ref }} / m-1$.

In this functional form, $F(p)$ is whatever aspect of the spectrograph we wish to model. In the specific example of GHOST, it will be the $x$-position (defined in the spatial direction) of each order in the first instance, but this same method is then used for the wavelength scale, and all three aspects of the slit image on the chip (spatial direction magnification scale, spectral direction magnification scale and rotation). All of these aspects are expected to change as a function of order, and position along the order.

This means that the simplest wavelength-scale spectrograph model should have:

- $q_{00}$ : central wavelength of order $m_{r e f}$;

- $q_{01}$ : central wavelength of order $m_{r e f}$;

- $q_{10}$ : central wavelength $/ R_{p i x}$, with $R_{p i x}$ the resolving power / pixel;

- $q_{11}$ : central wavelength $/ R_{p i x}$, with $R_{p i x}$ the resolving power / pixel;

with everything else approximately zero.

Note that the order of polynomials is left undefined. The code that handles these parameters is identical and left generalised since each aspect ( $x$-position, wavelength, etc) may require a different number of variables to fully describe the spectrograph. 
Table 2. Output options for the GHOSTDR Pipeline.

\begin{tabular}{|c|c|c|}
\hline Flag & Data Product & Description \\
\hline default & Default output & $\begin{array}{l}\text { Extracted, fully-processed object(s) and sky spectra. } \\
\text { Includes variance and data quality planes. Both order- } \\
\text { by-order and interpolated, single-spectrum data are pro- } \\
\text { vided. }\end{array}$ \\
\hline processed_image & Processed science image & $\begin{array}{l}\text { The final, processed science image, i.e. the state the } \\
\text { science data were in immediately prior to profile extrac- } \\
\text { tion. }\end{array}$ \\
\hline flat_profile & Extracted flat profile & $\begin{array}{l}\text { The flat-field profile used during data flat-field correc- } \\
\text { tion. }\end{array}$ \\
\hline sensitivity_curve & Calculated sensitivity curve & $\begin{array}{l}\text { The sensitivity curve (counts per unit of input flux as } \\
\text { a function of wavelength), determined during response } \\
\text { correction. This option will not be available if response } \\
\text { correction is not performed. }\end{array}$ \\
\hline
\end{tabular}

\subsubsection{Description of model file contents}

In the case of the $x$-position, using default file xmod.fits, the contents of this file are as follows:

$$
X_{\text {mod }}=\left[\begin{array}{lllll}
q_{24} & q_{23} & q_{22} & q_{21} & q_{20} \\
q_{14} & q_{13} & q_{12} & q_{11} & q_{10} \\
q_{04} & q_{03} & q_{02} & q_{01} & q_{00}
\end{array}\right]
$$

This non-standard way to define the variables within the files and imported array is related to the way numpys poly1d function takes inputs, with the highest-order coefficient first.

In the case of $x$-position, the coefficients represent:

- $q_{00}: x$ position of the middle of the reference order;

- $q_{01}$ : linear term coefficient for order spacing;

- $q_{02}$ : quadratic term coefficient for order spacing;

- $q_{10}$ : common rotation term for all orders;

- $q_{11}$ : linear term coefficient for order rotation;

- $q_{12}$ : quadratic term coefficient for order rotation;

- $q_{20}$ : common curvature term for all orders;

- $q_{21}$ : linear term coefficient for order curvature;

- $q_{22}:$ quadratic term coefficient for order curvature;

with everything else approximately zero.

\subsection{Pipeline Outputs}

The final primitive called on GHOST science data, formatOutput, provides multiple options for the data products that can be provided in the final output. These are summarised in Table 2. In order to preserve the ordering of data products in the final output bundle, these options are sequential (i.e., going down the table, each option will also provide all the output products from earlier options). 


\section{DISCUSSION AND FURTHER WORK}

At the time of publication, the data reduction software was not quite complete and ready for algorithm tuning and testing. Over the period July to December 2018, we anticipate testing and refinement of the data reduction software by the GHOST science team. The pipeline will be used during final integration activities at NRC Herzberg from January 2019, and final tweaks will be made during commissioning during the second half of 2019. When the software is used by scientists, we anticipate a small number of key challenges that are beyond the scope of the core data reduction effort. Firstly, the optimal algorithm for sky subtraction will often require a model of the sky emission. This is because shot noise from the sky will rarely dominate the error budget, and fewer fibers are used to measure sky than to measure object flux. In time, analysis of archival data will likely be used to create empirical models of sky emission for signal-to-noise optimal sky subtraction. The precision radial velocity mode of GHOST has the potential to be very powerful for sensitive follow-up of transiting planets, especially around cooler stars, as GHOST is expected to have both a higher sensitivity and longer wavelength coverage than the ESPRESSO instrument. ${ }^{3}$ Although GHOST has a fiber agitator, it has no fiber scramber due to the focus on high efficiency. This means that precision radial velocity at the $\sim 1 \mathrm{~ms}^{-1}$ level will at least require monitoring of the accross-slit measured fiber profile, and correction of the 2-dimensional extraction weights should the slit profile not be stable at the $10^{-3}$ pixel level.

\section{ACKNOWLEDGMENTS}

M. Ireland was partly supported by an Australian Research Council Future Fellowship (FT130100235).

\section{REFERENCES}

[1] Ireland, M., Anthony, A., Burley, G., Chisholm, E., Churilov, V., Dunn, J., Frost, G., Lawrence, J., Loop, D., McGregor, P., Martell, S., McConnachie, A., McDermid, R. M., Pazder, J., Reshetov, V., Robertson, J. G., Sheinis, A., Tims, J., Young, P., and Zhelem, R., "Progress on the Gemini High-Resolution Optical SpecTrograph (GHOST) design," in [Ground-based and Airborne Instrumentation for Astronomy V], Proc. SPIE 9147, 91471J (July 2014).

[2] Sharp, R. and Birchall, M. N., "Optimal Extraction of Fibre Optic Spectroscopy," PASA 27, 91-103 (Mar. 2010).

[3] Pepe, F. A., Cristiani, S., Rebolo Lopez, R., Santos, N. C., Amorim, A., Avila, G., Benz, W., Bonifacio, P., Cabral, A., Carvas, P., Cirami, R., Coelho, J., Comari, M., Coretti, I., De Caprio, V., Dekker, H., Delabre, B., Di Marcantonio, P., D’Odorico, V., Fleury, M., García, R., Herreros Linares, J. M., Hughes, I., Iwert, O., Lima, J., Lizon, J.-L., Lo Curto, G., Lovis, C., Manescau, A., Martins, C., Mégevand, D., Moitinho, A., Molaro, P., Monteiro, M., Monteiro, M., Pasquini, L., Mordasini, C., Queloz, D., Rasilla, J. L., Rebordão, J. M., Santana Tschudi, S., Santin, P., Sosnowska, D., Spanò, P., Tenegi, F., Udry, S., Vanzella, E., Viel, M., Zapatero Osorio, M. R., and Zerbi, F., "ESPRESSO: the Echelle spectrograph for rocky exoplanets and stable spectroscopic observations," in [Ground-based and Airborne Instrumentation for Astronomy III], 7735, 77350F (July 2010). 